\title{
STUDY ON THE INFLUENCE OF OPENING RESIDENTIAL QUARTER ON ROAD CAPACITY
}

\author{
Jing Tian \\ Department of Mathematics and Physics, North China Electric Power University, Baoding 071003, \\ China;
}

15932135705@163.com

\begin{abstract}
The concept of opening residential quarter has aroused the concern of all sectors of society. Influence on road capacity is a major focus of attention, including optimizing the road network structure, improving road capacity and improving traffic conditions. In this paper, in order to analyze the influence of opening residential on road capacity, an evaluation index system is established by collecting relevant data. This system mainly considers three indexes: the geographical factors of residential quarters, road condition, and signal facility. Based on this system, we build a fuzzy comprehensive evaluation model. By using this model, we can make reasonable proposals for the department of urban planning and traffic management from the point of view of improving the capacity of traffic.
\end{abstract}

Keywords: residential quarter, fuzzy comprehensive evaluation model

\section{Introduction}

The concept of opening residential quarter has aroused the concern of all sectors of society. We make research from the influence on road capacity. First, from the geographical factors of the community, we consider two sides: the size of the community area and the geographical location. The smaller of the size and the remoter of the location, the smaller of the influence on road capacity. Second, from the road condition, the road width is closely related to road capacity. The wider of the road, the bigger flow of traffic through the unit time, and the greater of road capacity. Third, because of the control of the signal light, the vehicles will be delayed at the intersection. The phenomenon is accumulated with time, which seriously affects the capacity of the road.

Finally, considering these three aspects, the fuzzy comprehensive evaluation model is established. By using this model, we can make reasonable proposals to the department of urban planning and traffic management from the point of view of improving the capacity of traffic.

\section{Evaluation index system}

\subsection{Geographical factors of the residential quarters}

We mainly consider the impact of the area and location of the residential quarters. When the area is smaller, the phenomenon of occupation will occur frequently. And then the phenomenon of traffic congestion will occur, which will causes certain traffic inconvenience. If the community is a 
suburb, vehicles are will fewer and road capacity is better. On the contrary, in the city center, road patency decreases seriously. The area and location are relatively speaking, so they are discussed only as qualitative indicators.

\subsection{Road conditions}

Lane width is an important factor to determine road capacity. Therefore, to meet the traffic demand, the road width must be calculated according to the actual demand. Here we only consider the width of a single lane of a motor vehicle. In city roads, most of the vehicles are small vehicles, and the width of the average value is $2.2 \mathrm{~m}$. By calculating the safety distance of the moving vehicles, the value of the two together is the width of a lane.

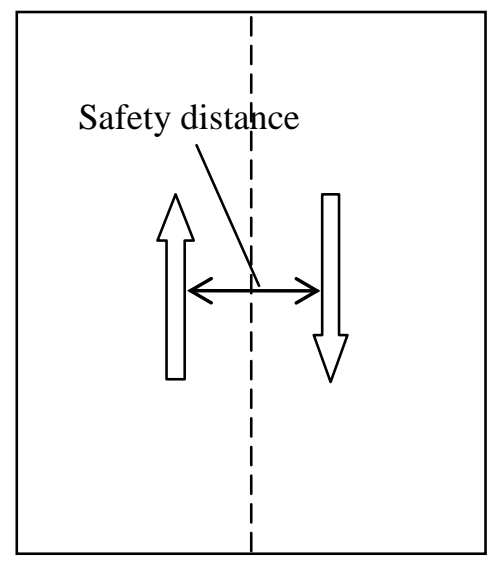

Fig.1 A single lane

The safety distance of the moving vehicles:

$$
x=0.7+0.02\left(V_{1}+V_{2}\right)^{3 / 4}
$$

$V_{1}, V_{2}$--average vehicle speed

The width of a single lane:

$$
y=2.2+x+1
$$

If the actual lane width is bigger than calculated lane width, the traffic congestion is serious. On the contrary, road capacity is good.

\subsection{Signal facilities}

From the point of view of the signal facilities, we mainly study the condition of the road outside the district before and after the opening. Outside the community, there are traffic lights. Due to the control of the signal light, there will be an average delay for vehicles in the intersection. However, for the district inside, there is no traffic lights. So we only consider the intersection delay in these two cases. We use TransModeler simulation to establish the mathematical model of two cases. 
Table 1 Symbols and Descriptions

\begin{tabular}{cl}
\hline Symbols & Descriptions \\
\hline$d_{1}$ & Average delay time of a vehicle at signalized intersection. \\
$C$ & Signal cycle length of traffic signal lights. \\
$u$ & Ratio of effective green time to cycle time within a signal period. \\
$a$ & Road saturation. \\
$G_{a l l}$ & The total time of green light in a cycle. \\
$R_{a l l}$ & The total time of red light in a cycle. \\
$d_{2}$ & Average delay time of a vehicle at unsignalized intersection. \\
$Q$ & Maximum vehicles per hour. \\
$T$ & Running time of traffic state. \\
\hline
\end{tabular}

1.with signal lights

The intersection delay model uses the TransModeler simulation software. The model is proposed on the base of ABBR timing control algorithm.

We consider the case of supersaturation:

$$
d_{1}=\frac{0.5 C(1-u)^{2}}{1-u a}
$$

$u$ : ratio of effective green time to cycle time within a signal period:

$$
u=\frac{G_{\text {all }}\left(C-R_{\text {all }}\right)}{C^{2}}
$$

The $d_{1}$ is smaller, and the vehicles delayed in the intersection are fewer.Thus road capacity is better.

\section{2.without signal lights}

There is no signal control in the road inside the community. When the community is open, people will choose relatively close and fast road. These no signal controlled intersections will be the first choice for people to walk.

$$
d_{2}=\frac{3600}{Q}+900 T\left[(a-1)+\sqrt{(a-1)^{2}+8 a /(Q T)}\right]+5
$$

The $d_{2}$ is smaller, and the vehicles delayed in the intersection are fewer.Thus road capacity is better.

\section{Fuzzy comprehensive evaluation}

According to the actual situation of road network system,the fuzzy comprehensive evaluation model is established from the point of view of representation and applicability.

The weight value of each evaluation index and the relative membership value of each corresponding evaluation index are multiplied and accumulated. Thus, we obtain the 
comprehensive index value of fuzzy evaluation. The greater the comprehensive index value, the more the influence of these factors on the whole. Based on it, we can make scientific decisions. Comprehensive index value:

$$
Z=\sum_{i=1}^{m} w_{i} r_{i}
$$

wi: the weight value of each evaluation index

ri: the relative membership value of each corresponding evaluation index

\section{Conclusions}

Through the above analysis, we can make reasonable proposals to the department of urban planning and traffic management from the point of view of improving the capacity of traffic.

First, the construction of the opening residential quarters should be integrated into the overall planning of the city. Second, add traffic lights. After the opening of the District, the road network density increases, which avoids people traveling at random, thus resulting in road congestion. Third, implement variable lane. In the morning and evening peak hour, traffic flow has obvious changes in the secondary sections. A certain number of lanes are only for a certain period of time in a particular direction. This will improve road capacity and reduce congestion.

\section{References}

[1] Qiang Shao,Youjun Li, Qingwang Tian, Construction method of comprehensive evaluation index system, J. Sci. Commun. 03(2004)74-76+105-123.

[2] Jvliang Jin, Yiming Wei, Jing Ding,Fuzzy comprehensive evaluation model based on Improved Analytic Hierarchy Process,J. Sci. Commun.03(2004)65-70.

[3] Zhangli Hu, Ping He, Ming Yang, Analysis of the influence of urban road lane width on traffic capacity,J. Sci. Commun. 01(2012)1-2+5. 